\title{
Consumo de sal y riesgo cardiovascular
}

\author{
Salt intake and cardiovascular risk
}

Strazzullo P y col. November 2009, doi:10.1136/bmj.b4567.

\section{Objetivo}

Evaluar la relación entre el nivel de consumo de sal habitual y el riesgo de padecer enfermedad cardiovascular (ECV) y/o cerebrovascular (ACV).

\section{Diseño y fuente de datos}

Meta-análisis de estudios prospectivos. Se realizó una búsqueda bibliográfica en Medline (1966-2008) EMBASE (desde 1988) AMED (desde 1985) CINAHL (desde 1982) Psychinfo (desde 1985) y Biblioteca Cochrane.

\section{Selección de estudios}

Fueron identificadas 3246 publicaciones, de las cuales 13 cumplieron con los criterios de inclusión: estudios poblacionales prospectivos de tres o más años de seguimiento publicados entre 1966 y 2008, que hubieran evaluado en adultos y desde su inicio, la exposición a la ingesta de sal y determinado la incidencia de ACV o ECV como resultado y el tipo o número de eventos en las diferentes subgrupos divididos de acuerdo a la magnitud de su ingesta de sal.

\section{Extracción de datos}

Fue llevada a cabo por dos revisores independientes. Si había discrepancias sobre la inclusión de estudios o la interpretación de los datos, dos árbitros decidían si se lo incluía o no. Si faltaba información, se contactaba a los autores y se les solicitaba la información necesaria. Se extrajeron los datos sobre riesgos relativos e intervalos de confianza de cada estudio y se reunieron siguiendo un modelo de efecto aleatorio, ponderando la inversa de la varianza. Se realizó un análisis de la heterogeneidad, de sesgo de publicación, de subgrupo y de metaregresión.

\section{Resultados Principales}

Fueron incluidos 13 estudios que informaron sobre 19 cohortes independientes con una población total de 177.025 participantes. El promedio del seguimiento osciló entre 3,5 y 19 años. Fueron reportados 10.507 eventos (5.346 ACV y 5.161 ECV).

Tabla 1: riesgo de enfermedad cardio y cerebrovascular de acuerdo a la magnitud de consumo de sal comparando entre subgrupos con una diferencia de consumo de sal de seis gramos y asignando un riesgo relativo igual a uno $(R R=1)$ al subgrupo de menor consumo.

\begin{tabular}{c|c}
\hline Resultado evaluado & Riesgo relativo e IC95\% \\
\hline Accidente cerebrovascular & $1,55(1,20$ a 2,00$)$ \\
\hline Enfermedad cardiovascular & $1,14(0,99$ a 1,31$)$ \\
\hline
\end{tabular}

IC 95\%: Intervalo de confianza del 95\%.

\section{Conclusiones}

El resultado de éste meta-análisis proporciona evidencia de una la asociación directa entre la ingesta elevada de sal en la dieta, el riesgo de ACV y de ECV. A pesar de la gran heterogeneidad entre las 14 cohortes, los resultados se ven reforzados por la falta de sesgos de publicación, apoyando la hipótesis de que una reducción del consumo de sal a nivel poblacional puede ayudar a la prevención de eventos cardiovasculares.

Palabras claves: consumo de sal, accidente cerebrovascular, enfermedad cardiovascular, estudios prospectivos.

Key words: salt intake, stroke, cardiovascular disease, prospective studies.

Fuente de financiamiento: EC Grant (FP7-HEALTH-2007-201550).

\section{Comentario}

Las enfermedades cardiovasculares son la principal causa de muerte en los mayores de 60 años y la segunda, entre los 15 y los 59. Según la Organización Mundial de la Salud (OMS) el 62\% de todos los ACV y el $49 \%$ de los ECV son atribuibles a la hipertensión arterial ${ }^{1}$. Conociéndose la asociación entre los niveles de consumo de sal en la dieta y los niveles de TA, es razonable esperar una reducción considerable en la incidencia de ACV y de ECV, consecutiva a la reducción en la ingesta de sal. Se recomienda que el promedio de consumo de sal diario sea inferior a cinco o seis gramos. Sin embargo, la mayoría de los adultos consume diariamente un promedio superior a seis gramos, superando en muchos países los 12 (por ejemplo Argentina y España).

Ensayos clínicos controlados aleatorizados demostraron que es posible conseguir una reducción significativa de la tensión arterial (TA) con una dieta reducida en sodio (seis gramos diarios) lo que genera una reducción de 4 a $7 \mathrm{mmHg}$ en hipertensos y de 2 a $4 \mathrm{mmHg}$ en normotensos ${ }^{2}$.

Éste meta-análisis aporta evidencia sobre la asociación entre el consumo de sal en la dieta y el riesgo de ACV y la ECV. Sin embargo, presenta una limitante importante ya que los estudios incluidos son muy heterogéneos (tamaño muestral, número de eventos, duración del seguimiento y forma de registrar el consumo de sal). Respecto de este último punto, la estimación de la ingesta de sal de la población de referencia en cada estudio se basó en una sola medición (ya sea a través de la natriuria de 24 horas o de encuestas sobre el consumo de sal en la dieta) por lo que las estimaciones del riego pueden estar subregistradas, ya que la ingesta de sal es muy variable.

\section{Conclusiones de la comentadora}

Existen experiencias como la llevada a cabo en el Reino Unido de Gran Bretaña ${ }^{3}$, que lograron reducir el contenido en sal en alimentos procesados entre un $20 \%$ y un $30 \%$. A pesar de sus limitaciones, los resultados del estudio que hemos comentado podrían servir para impulsar estrategias en salud pública destinadas a disminuir la cantidad de sal en los alimentos procesados ${ }^{4}$.

Buela Gabriela [ Servicio de Medicina Familiar y Comunitaria, Hospital Italiano de Buenos Aires. gabriela.buela@hospitalitaliano.org.ar ]

Buela G. Consumo de sal y enfermedad cardiovascular. Evid Act Pract Ambul. Vol 13 (2) 55. Abr-Jun 2010. Comentado de: Strazzullo P y col. Salt intake, stroke, and cardiovascular disease: meta-analysis of prospective studies. November 2009, doi:10.1136/bmj.b4567. PMID: 19934192.

\section{Referencias}

1. World Health Organization. The world health report 2002-reducing risks promoting healthy life. WHO, 2002.

2. He F y col. Effect of modest salt reduction on blood pressure: a meta-analysis of randomized trials. Implications for public health. J Hum Hypertens $2002 ; 16: 761-70$.

3. Murray C y col. Effectiveness and costs of interventions to lower systolic blood pressure and cholesterol: a global and regional analysis on reduction of cardiovascular-dis3. Murray C y col. Effectiveness and

ease risk. Lancet 2003;361:717-25.
4. Selmer R y col.Cost and health consequences of reducing the population intake of salt. J Epidemiol Community Health 2000;54:697-702. 